SUPPORTING INFORMATION

\title{
An Extensive Test of Fourteen Scoring Functions Using the PDBbind Refined Set of 800 Protein-Ligand Complexes
}

Renxiao Wang, Yipin Lu, Xueliang Fang, and Shaomeng Wang*

Department of Internal Medicine and Comprehensive Cancer Center, University of Michigan Medical School and Department of Medicinal Chemistry, University of Michigan College of

Pharmacy, 3316 CCGC Building, 1500 E. Medical Center Drive, Ann Arbor, MI 48109-0934, U.S.A. 
The fourteen scoring functions tested in our study are briefly described below, including X-Score (version 1.1), DrugScore (version 1.2), five scoring functions from the CSCORE module in the Sybyl software (version 6.9), four scoring functions from the Ligand Scoring module in the Cerius2 software (version 4.6), two scoring functions from the GOLD program (version 2.1), and the HINT scoring function (version 3.06). Details of these scoring functions can be found in the cited references. To facilitate readers' better assessment and reproduction of our results, the key parameters and settings used in our application of these scoring functions are also given.

X-Score. This empirical scoring function was developed in our group. ${ }^{1} \mathrm{X}$-Score combines terms for van der Waals interaction, hydrogen bonding, hydrophobic effect and deformation effect, and can be expressed as:

$$
\Delta G_{\text {binding }}=\Delta G_{v d w}+\Delta G_{H-\text { bond }}+\Delta G_{\text {hydrophotic }}+\Delta G_{\text {rotor }}+\Delta G_{0}
$$

This version of X-Score provides three variations, which differ from each other in the hydrophobic effect term: HPScore computes this term by counting the hydrophobic atom pairs formed between the protein and ligand molecules; HMScore computes this term by examining the match of hydrophobic ligand atoms into hydrophobic environments; HSScore computes this term with buried hydrophobic surface area upon binding. The X-Score program requires the protein in PDB format and the ligand in Mol2-format.

DrugScore. This scoring function is based on knowledge-based potentials. ${ }^{2,3}$ The total protein-ligand binding score is expressed as a combination of distance-dependent potentials and surface-dependent potentials: 


$$
\Delta W=\gamma \times \sum_{\text {protein ligand }} \sum_{i, j} \Delta W_{i}(r)+(1-\gamma) \times\left[\sum_{\text {ligand }} \Delta W_{i}\left(S A S, S A S_{0}\right)+\sum_{\text {protein }} \Delta W_{j}\left(S A S, S A S_{0}\right)\right]
$$

The DrugScore program evaluated in this study was obtained directly from its authors. It provides three variations for calculating protein-ligand binding scores, which uses either pure pairwise potentials, pure surface-dependent potentials, or a combination of both as indicated in the above equation. These three variations are referred as DrugScore::Pair, DrugScore::Surf, and DrugScore::Pair/Surf. The DrugScore program requires the protein in PDB format and the ligand in Mol2 format.

Sybyl. Five scoring functions are implemented in the CScore module in the Sybyl software. $^{4}$ They are referred as Sybyl::D-Score, Sybyl::PMF-Score, Sybyl::G-Score, Sybyl::ChemScore, and Sybyl::F-Score in this paper. In our study, both protein and ligand molecules were loaded into Sybyl in Mol2-format files. No structural optimization on either the ligand or the protein was performed before binding score computation. If not specified below, default parameters provided by Sybyl were used in the application of these scoring functions.

(1) Sybyl::D-Score. This scoring function originates from the molecular docking program DOCK. ${ }^{5}$ It is a classical force field energy function, which sums van der Waals and electrostatic interactions between the complex:

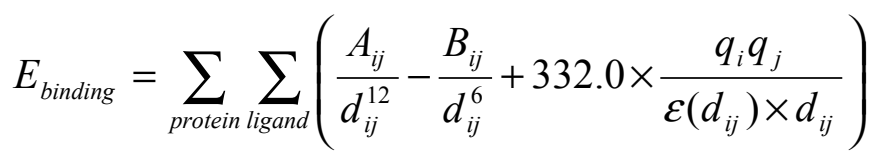

In our computation, the van der Waals energy was calculated using the Tripos force field. Hydrogen atoms were assigned a small radius of $1.0 \AA$. A ceiling of $0.5 \mathrm{kcal} / \mathrm{mol}$ was set to any van der Waals pair to prevent outbreak of repulsion energies. To compute the electrostatic energy, protein atoms were assigned AMBER all-atom charges, and ligand atoms were assigned 
MMFF94 charges. Distance-dependent dielectric constant was adopted in the electrostatic term. A distance cutoff of $12 \AA$ was applied to both of the van der Waals and the electrostatic term.

(2) Sybyl::PMF-Score. This potential of mean force scoring function is based on the work of Muegge et al. ${ }^{6-8}$ The protein-ligand interaction energy is calculated as a sum of distancedependent pairwise potentials over all heavy atom pairs between the complex. Both enthalpic and entropic effects are assumed to be included implicitly:

$$
P M F=\sum_{\text {protein ligand }} A_{i j}\left(d_{i j}\right)
$$

According to the authors' original publication, a distance cutoff of $6 \AA$ for carbon-carbon atom pairs and a cutoff of $9 \AA$ for all the other atom pairs were used in our computation.

(3) Sybyl::G-Score. This is Sybyl's implementation of the GoldScore scoring function from the GOLD program. ${ }^{9,10}$ It is basically a force field based scoring function, which is the sum of an external van der Waals term, an external hydrogen-bond term, and an internal strain energy term:

$$
\begin{aligned}
E_{\text {total }} & =E_{\text {vdw }, \text { ext }}+E_{H-\text { bond, ext }}+E_{\text {strain, int }} \\
& =\sum_{\text {protein ligand }} \sum_{i j}\left(\frac{A_{i j}}{d_{i j}^{8}}-\frac{B_{i j}}{d_{i j}^{4}}\right)+\sum_{\text {protein ligand }} \sum_{d a}\left[\left(E_{d w}+E_{w w}\right)-\left(E_{d w}+E_{a w}\right)\right] \\
& +\left\{\sum_{\text {ligand }}\left(\frac{C_{i j}}{d_{i j}^{12}}-\frac{D_{i j}}{d_{i j}^{6}}\right)+\sum_{\text {ligand }} \frac{1}{2} V\left[1+\frac{n}{|n|} \cos (|n| \cdot \omega)\right]\right\}
\end{aligned}
$$

Both of the external van der Waals energy and the internal strain energy are calculated with the parameters in the Tripos force field. In our computation, the ceiling of any van der Waals pair was also set to $0.5 \mathrm{kcal} / \mathrm{mol}$. A distance cutoff of $9 \AA, 7 \AA$, and $12 \AA$ was assigned to the external van der Waals energy term, the external hydrogen bonding term, and the internal strain term, respectively. 
(4) Sybyl::ChemScore. This empirical scoring function is based on the work of Eldridge et al. ${ }^{11,12}$ which has a hydrogen-bond term, a coordinate bond term concerning metal ions, a hydrophobic effect term, and a "rotor" term:

$$
\begin{aligned}
\Delta G_{\text {binding }}= & \Delta G_{H-\text { bond }} \sum_{H-\text { bond }} f(\Delta R, \Delta \alpha)+\Delta G_{\text {metal }} \sum_{\text {metal }} f(\Delta R, \Delta \alpha) \\
& +\Delta G_{\text {lipo }} \sum_{\text {lipo }} f(\Delta R)+\Delta G_{\text {rotor }} \sum_{\text {rotor }} f\left(P_{n l}, P_{n l}^{\prime}\right)+\Delta G_{0}
\end{aligned}
$$

(5) Sybyl::F-Score. This empirical scoring function was originally implemented in the molecular docking program FlexX..$^{13}$ It is actually a variation of the LUDI scoring function ${ }^{14}$ and is specially optimized for molecular docking tasks:

$$
\begin{aligned}
\Delta G_{\text {binding }} & =\Delta G_{H-\text { bond }} \sum_{H-\text {-bond }} f(\Delta R, \Delta \alpha)+\Delta G_{\text {ionic }} \sum_{\text {ionic }} f(\Delta R, \Delta \alpha)+\Delta G_{\text {aromatic }} \sum_{\text {aromatic }} f(\Delta R, \Delta \alpha) \\
& +\Delta G_{\text {contact }} \sum_{\text {contact }} f(\Delta R)+\Delta G_{\text {rotor }} \times N_{\text {rotor }}+\Delta G_{0}
\end{aligned}
$$

It sums neutral hydrogen bonds, ionic hydrogen bonds (salt bridges), hydrophobic interactions between aromatic groups, general contacts formed between the complex, and a "rotors" term for the ligand molecule.

Cerius2. Four scoring functions are implemented in the Ligand Scoring module in the Cerius2 software. ${ }^{16}$ They are referred as Cerius2::LigScore, Cerius2::PLP, Cerius2::PMF, and Cerius2::LUDI in this paper. In our study, the protein molecules were loaded into Cerius2 in PDB format; while the ligand molecules were loaded in SD format. No structural optimization of either the ligand structure or the protein structure was performed before binding score computation.

(1) Cerius2::LigScore. As for as we know, the only locatable description of this scoring function is in the Cerius2 user manual. It consists of a sum of a softened Lennard-Jones 6-9 van der Waals potential, a count of the buried polar surface area between the complex involving 
attractive protein-ligand interactions, and a square of the buried polar surface area between the complex involving both attractive and repulsive protein-ligand interactions:

$$
p K_{i}=A-B \times v d W+C \times C_{+p o l}-D \times \text { Totpol }^{2}
$$

It is therefore an empirical scoring function. We chose the CFF force field parameters with the "exact pairwise" option in our work. For the convenience in computation, the entire protein molecule was defined as the binding site.

(2) Cerius2::PLP. This empirical scoring function ${ }^{17,18}$ is a sum of pairwise linear potentials between ligand and protein heavy atoms with parameters dependent on interaction type:

$$
E_{\text {total }}=E_{H-\text { bond }}+E_{\text {repulsion }}+E_{\text {contact }}
$$

Each interacting atom pair is assigned one of the three interaction types: hydrogen bonding between donors and acceptors, repulsive donor-donor and acceptor-acceptor contacts, and generic dispersion of other contacts. In Cerius2, there are two variations of this scoring function, namely PLP1 and PLP2. The Cerius2 user manual mentions that they employ slightly different algorithms and parameters sets without further details.

(3) Cerius2::PMF. This is Cerius2' implementation of the PMF-Score developed by Muegge et al..$^{6-8}$ The same $6 \AA / 9 \AA$ distance cutoff was used in our computation.

(4) Cerius2::LUDI. This scoring function was originally developed by Böhm ${ }^{14,15}$ and is one of the pioneering empirical scoring functions. It computes protein-ligand binding affinity as a sum of neutral and ionic hydrogen bonds formed between the complex, hydrophobic surface area buried upon binding, and "rotors" of the ligand molecule: 


$$
\begin{aligned}
\Delta G_{\text {bind }}= & \Delta G_{H-\text { bond }} \sum_{H-\text { bond }} f(\Delta R, \Delta \alpha)+\Delta G_{\text {ionic }} \sum_{\text {ionic }} f(\Delta R, \Delta \alpha) \\
& +\Delta G_{\text {hydrophobic }} \sum_{\text {hydrophobic }}\left|A_{\text {hydrophobic }}\right|+\Delta G_{\text {rotor }} \times N_{\text {rotor }}+\Delta G_{0}
\end{aligned}
$$

Cerius2 offers three different implementations of this scoring function, namely LUDI1, LUDI2, and LUDI3. Again, the Cerius2 user manual does not detail the difference between them.

GOLD. GOLD is a molecular docking program ${ }^{9,10}$ distributed by the Cambridge Crystallographic Data Centre (http://www.ccdc.cam.ac.uk/). This program has implemented GoldScore and ChemScore as its internal scoring functions, which are referred as GOLD::GoldScore and GOLD::ChemScore in this paper. In our computation, we loaded the protein molecules in PDB format and the ligand molecules in Mol2 format into GOLD. If not specified below, default settings given in the GOLD configuration file were used in our computation.

(1) GOLD::GoldScore. The basics of this scoring function have been described earlier in this paper. In the GOLD program, GoldScore by default is computed as:

$$
\text { GoldScore }=1.375 \times V D W_{\text {external }}+H B_{\text {external }} \dashv S E_{\text {internal }}
$$

Here, $V D W_{\text {external }}$ is the van der Waal score between the protein and the ligand; $H B_{\text {external }}$ is the hydrogen bonding score between the complex; while $S E_{\text {internal }}$ denotes for the internal strain energy (including van der Waals and torsional factors) of the ligand molecule. The empirical weight factor of 1.375 is applied to the external van der Waals term to compensate hydrophobic contacts between the complex since GoldScore does not have an explicit term for such interactions.

(2) GOLD::ChemScore. This is GOLD's implementation of the ChemScore scoring function, ${ }^{11,12}$ which has also been described earlier in this paper. However, GOLD has its own 
modifications: the final score is obtained by adding clash penalties and an internal torsion term, which militate against bad contacts in docking and poor conformations.

$$
G O L D:: \text { ChemScore }=\text { ChemScore }+P_{\text {clash }}+P_{\text {internal }}
$$

Finally, in our study the GOLD program was used only to score a given protein-ligand complex in place without altering the coordinates of either the ligand or the protein. For this purpose, the "optimize_flag $=$ LOCAL" option in the GOLD configuration file was always enabled in our computation, which restricted the program not to adjust the coordinates of any atom except "rotatable" hydrogen atoms. Another parameter in the GOLD parameter file, namely "do_simplex", controls whether a quick local optimization of the ligand conformation shall be performed before the binding score is computed. To make a comparison, we also computed another set of binding scores by using GoldScore and ChemScore with this option was enabled. To distinguish from the results computed directly on crystal structures, the results produced from this procedure are referred as GOLD::GoldScore_opt and GOLD::ChemScore_opt in this paper.

HINT. The HINT (Hydropathic INTeractions) scoring function tested in our study is implemented in the HINT program, which is distributed by the EduSoft Inc. (http://www.eslc.vabiotech.com/). HINT was originally developed as a tool for computing hydrophobic field in 3D-QSAR studies, but recently it has been extended to compute proteinligand interaction energies. ${ }^{19}$ In brief, the HINT score is a pairwise summation of "hydropathic" interactions between the protein and the ligand.

$$
H I N T=\sum_{\text {protein ligand }} \sum_{i j}=\sum_{\text {protein ligand }} \sum_{i} a_{i} S_{j} a_{j} R_{i j} T_{i j}
$$

In this equation, $b_{i j}$ is the contribution from the interaction between protein atom $i$ and ligand atom $j ; S_{i}$ and $S_{j}$ are solvent accessible surface areas; $a_{i}$ and $a_{j}$ are hydrophobic atom constants, which are derived from partitioned $\log P$ values; $R_{i j}$ is a distance-dependent weight factor of the 
interaction between $i$ and $j$; while $T_{i j}$ is a discriminant function designed to keep the signs of interactions consistent with the HINT convention that favorable interactions are positive and unfavorable interactions are negative. 


\section{REFERENCES}

1. Wang, R.; Lai, L. \& Wang, S. Further development and validation of empirical scoring functions for structure-based binding affinity prediction. J. Comput.-Aided Mol. Des. 2002, $16,11-26$.

2. Gohlke, H.; Hendlich, M. \& Klebe, G. Knowledge-based scoring function to predict proteinligand interactions. J. Mol. Biol. 2000, 295, 337-356.

3. Gohlke, H.; Hendlich, M. \& Klebe, G. Predicting binding modes, binding affinities and "hot spots" for Protein-ligand complexes using a knowledge-based scoring function. Perspectives in drug discovery and design, 2000, 20, 115-144.

4. The SYBYL software, version 6.9, Tripos Inc., http://www.tripos.com/.

5. Ewing, T. J. A.; Makino, S.; Skillman, A. G.; Kuntz, I. D. DOCK 4.0: Search strategies for automated molecular docking of flexible molecule databases. J. Comput.-Aided Mol. Des. 2001, 15, 411-428.

6. Muegge, I. \& Martin, Y. C. A general and fast scoring function for protein-ligand interactions: A simplified potential approach. J. Med. Chem. 1999, 42, 791-804.

7. Muegge, I. A knowledge-based scoring function for protein-ligand interactions: Probing the reference state. Perspectives in Drug Discovery \& Design, 2000, 20, 99-114.

8. Muegge, I. Effect of ligand volume correction on PMF scoring. J. Comp. Chem. 2001, 22, 418-425.

9. Jones, G.; Willett, P.; Glen, R.C.; Leach, A.R.; Taylor, R. Development and validation of a genetic algorithm for flexible docking. J. Mol. Biol. 1997, 267, 727-748. 
10. Jones, G.; Willett, P.; Glen, R.C. Molecular recognition of receptor sites using a genetic algorithm with a description of desolvation. J. Mol. Biol. 1995, 245, 43-53.

11. Eldridge, M. D.; Murray, C. W.; Auton, T. R.; Paolini, G. V. \& Mee, R. P. Empirical scoring functions: I. The development of a fast empirical scoring function to estimate the binding affinity of ligands in receptor complexes, J. Comput.-Aided Mol. Des. 1997, 11, 425-445.

12. Murray, C. W.; Auton, T. R. \& Eldridge, M. D. Empirical scoring functions. II. The testing of an empirical scoring function for the prediction of ligand-receptor binding affinities and the use of Bayesian regression to improve the quality of the model. J. Comp.-Aided Mol. Des. 1998, 12, 503-519.

13. Rarey, M.; Kramer, B.; Lengauer, T.; Klebe, G. A fast flexible docking method using an incremental construction algorithm. J. Mol. Biol. 1996, 261, 470-489.

14. Böhm, H. J. The development of a simple empirical scoring function to estimate the binding constant for a protein-ligand complex of known three-dimensional structure, J. Comp.-Aided Mol. Des. 1994, 8, 243-256.

15. Böhm, H. J. Prediction of binding constants of protein ligands: A fast method for the prioritization of hits obtained from de novo design or 3D database search programs, $J$. Comp.-Aided Mol. Des. 1998, 12, 309-323.

16. The Cerius2 software, version 4.6, Accelrys Inc., http://www.accelrys.com/.

17. Verkhivker, G.; Appelt, K.; Freer, S.T.; Villafranca, J.E. Empirical free energy calculations of ligand-protein crystallographic complexes. I. Knowledge-based ligand-protein interaction potentials applied to the prediction of human immunodeficiency virus 1 protease binding affinity. Protein Eng. 1995, 8, 677-691. 
18. Verkhivker, G. M.; Bouzida, D.; Gehlhaar, D. K.; Rejto, P. A.; Arthurs, S.; Rose, P. W. Deciphering common failures in molecular docking of ligand-protein complexes. J.Comput.Aided Mol.Des. 2000, 14, 731-751.

19. Cozzini, P.; Fornabaio, M.; Marabotti, A.; Abraham, D. J.; Kellogg, G. E. \& Mozzarelli, A. Simple, Intuitive Calculations of Free Energy of Binding for Protein-Ligand Complexes. 1. Models without Explicit Constrained Water. J. Med. Chem. 2002, 45, 2469-2483. 